\title{
TUMOR DE CORPO CAROTÍDEO: RELATO DE 3 CASOS
}

\section{CAROTID BODY TUMOR: REPORT OF 3 CASES}

\author{
Hussein El Zein ${ }^{*}$, Daniel S Ferruzzi ${ }^{1}$, Jeferson F Toregeani ${ }^{2}$ \\ ${ }^{1}$ Acadêmico do Medicina do Centro Universitário da Fundação Assis Gurgacz. ${ }^{2}$ Médico, Mestre. \\ Professor das disciplinas de Fisiologia Humana, Angiologia e Cirurgia Vascular no curso de Medicina \\ do Centro Universitário da Fundação Assis Gurgacz
}

*Autor correspondente: husseinelzein_@hotmail.com. ORCID: https://orcid.org/0000-0002-0173-3551

DOI: 10.35984/fjh.v2i1.148

RESUMO

O tumor de corpo carotídeo é uma neoplasia rara de caráter predominantemente benigno que afeta o glomus carotídeo, mais comumente em pessoas de meia idade, mas também crianças. Sua apresentação clínica é variável, desde formas assintomáticas até paralisia de nervos cranianos. Ao exame físico pode ser encontrado apenas elevação de região cervical no triângulo do pescoço. O diagnóstico é confirmado por meio de exames de imagens e o tratamento de escolha é a remoção cirúrgica. Este relato aborda três pacientes diagnosticados com tumor de seio carotídeo e acompanhados na cidade de Cascavel-PR.

Palavras-chave: glomus carotídeo; seio carotídeo; paraganglioma.

\section{ABSTRACT}

The carotid body tumor is a rare neoplasm of predominantly benign character that affects carotid glomus, most commonly in middle-aged people, but also in children. Its clinical presentation is variable, from asymptomatic forms to paralysis of cranial nerves. On physical examination, only elevation of the cervical region in the neck triangle can be found. The diagnosis is confirmed by means of imaging examinations and the treatment of choice is surgical removal. This report approaches three patients diagnosed with carotid sinus tumor and followed up in the city of Cascavel-PR.

Keywords: carotid body; carotid glomus; chemodectoma.

\section{INTRODUÇÃO}

O corpo carotídeo, glomo carotídeo ou glomus carotídeo, é uma estrutura elíptica de aproximadamente $3-4 \mathrm{~mm}$ formada pelo agrupamento de quimiorreceptores, localizada na bifurcação da carótida comum bilateralmente. É ricamente irrigado por capilares fenestrados, inervado por ramos sensitivos do IX nervo craniano e possui função de controle homeostático, visto que apresenta receptores sensíveis ao $\mathrm{pH}$, concentração sérica de dióxido de carbono e oxigênio (SOUSA et al, 2000; VIEIRA et al, 2009; MESQUITA et al, 2016).

O tumor de corpo carotídeo (TUCC), muitas vezes trazido como paraganglioma pela literatura, é raro, podendo ocorrer em crianças, mas geralmente acomete indivíduos de meia idade (45 anos) e benigno em $90-95 \%$ dos casos. Três diferentes tipos de TUCC são descritos na literatura: familiar, esporádico e hiperplásico. Sendo o tipo esporádico responsável por aproximadamente $85 \%$ dos casos (SAJID et al, 2007). 


\section{METODOLOGIA}

O presente trabalho se encontra em cumprimento com a Resolução 196/96 do Conselho Nacional de Saúde, visto se tratar de pesquisa com ser humano. Previamente à sua realização, este foi enviado ao Comitê de Ética e Pesquisa do Centro Universitário da Fundação Assis Gurgacz e aprovado pelo número CAAE 14382619.9.0000.5219. O estudo é do tipo relato de caso em série e constitui-se em uma pesquisa fenomenológica e descritiva, com abordagem qualitativa e transversal. Será realizada através da análise do quadro clínico de três pacientes com diagnóstico de paraganglioma. A pesquisa documental terá por base o prontuário clínico dos pacientes e exames complementares.

\section{RELATO DE CASO}

O primeiro paciente, 25 anos, sexo masculino, branco, estudante, natural de Laranjeiras do Sul (PR) e residente em Cascavel (PR), procurou atendimento médico já com objetivo de intervenção cirúrgica após aparecimento de nódulo em região cervical esquerda notado há 1 ano, associado apenas a dores esporádicas. Negava sintomas como odinofagia, disfagia e disfonia. Ao exame físico foi constatado nódulo de consistência endurecida com presença de edema, sem alterações neurológicas ou em cavidade oral. Foi então solicitado angiotomografia computadorizada (Figura 1) como pré-operatório e encaminhado para procedimento cirúrgico. Após cinco meses do primeiro atendimento foi realizada cirurgia com implantação de ponte Carot-Carot e estudo histológico e perfil imunohistoquímico que condizia com paraganglioma (tumor gômico). Paciente evoluiu no pós-operatório com paralisia de corda vocal esquerda realizando então acompanhamento com otorrinolaringologia e fonoaudiologia. Solicitado ultrassonografia com doppler (ecodoppler) de carótidas para controle, não foi evidenciado qualquer tipo de alteração ou anormalidade. Paciente evoluiu bem clinicamente e encontra-se em seguimento ambulatorial de forma anual.

A segunda paciente, 45 anos, sexo feminino, branca, do lar, natural de Guaíra (PR) e residente em Cascavel (PR), também procurou atendimento médico com intuito de retirada cirúrgica de nódulo que surgiu em região cervical há um ano. Negava sintomas como disfagia e odinofagia. Ao exame físico foi constatado nódulo endurecido, indolor a palpação, sem alterações neurológicas ou em cavidade oral. Após conversa com paciente e familiares sobre os riscos cirúrgicos foi solicitado exames pré-operatórios. Após nove meses do primeiro atendimento foi realizada cirurgia com novo ecodoppler para mapeamento da lesão que mostrou dimensões de $2,91 \mathrm{~cm} \times 4,84 \mathrm{~cm}$ atingindo o ângulo da mandíbula. Paciente apresentou boa evolução no pós-operatório, acompanhamento com fonoaudiologia e melhora da disfagia; atualmente faz acompanhamento ambulatorial anualmente.

O terceiro paciente, 45 anos, sexo masculino, branco, comerciante, apresentava aumento progressivo em região cervical há três meses, sendo mais acentuado à direita e mais brando à esquerda, sem outros sintomas associados. Ao exame físico foi observado lesão nodular com dimensões endurecida, indolor a palpação; com ausência de alterações em nervos cranianos e cavidade oral. Com a suspeição de glômus carotídeo foi solicitado ressonância nuclear magnética e angiotomografia computadorizada. Após resultado dos exames de imagem foi 
agendado e realizado o procedimento cirúrgico em região cervical direita. Após um mês foi ressecado paraganglioma de seio carotídeo esquerdo. Realizado ecodoppler de carótidas para controle não foi evidenciado anormalidades. Paciente seguiu com boa evolução e ausência de alterações referentes à nervos cranianos e sem necessidade de acompanhamento de outras especialidades. Atualmente paciente realiza acompanhamento ambulatorial anual.

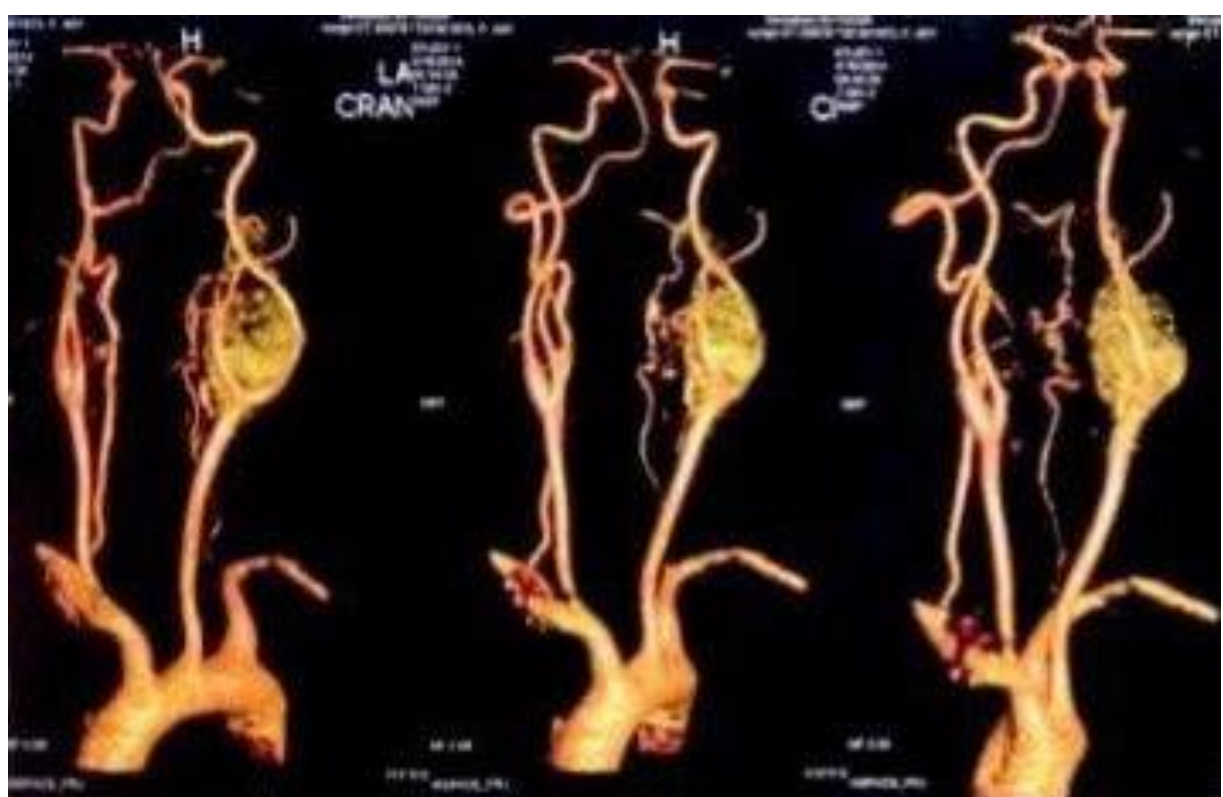

Figura 1 - Angiotomografia computadorizada pré-operatória Paciente 1. Fonte: prontuário do paciente.

\section{DISCUSSÃO}

O TUCC constitui cerca de $65 \%$ dos tumores de cabeça e pescoço e é considerado uma doença de meia idade, embora crianças também possam ser acometidas (KOTELIS et al, 2009). Classificado em três tipos principais conforme a literatura: familiar, esporádico e hiperplásico. Sendo o tipo esporádico responsável por 85\% dos casos de TUCC (SAJID, HAMILTON e BAKER, 2007); o mesmo tipo que se observa nos três pacientes descritos. Detém caráter benigno na maioria das vezes, mas pode apresentar caráter de malignidade que pode ser confirmada pela presença de metástase em linfonodos regionais, estruturas adjacentes e órgãos como fígado e pulmões (VEIRA et al, 2009), o que não foi encontrado nos pacientes descritos no caso.

A apresentação clínica e exame físico do TUCC é relativamente pobre, sendo que na maioria das vezes se apresenta apenas como uma massa palpável no triângulo anterior do pescoço e somente $10 \%$ dos casos cursam com alterações de nervos cranianos (ISIK et al, 2007). Conforme os casos descritos, nenhum dos pacientes apresentou paralisia de nervos cranianos na evolução natural da doença; os sintomas referentes à disfonia e disfagia apareceram após a intervenção cirúrgica.

Exames de imagens como ultrassonografia com doppler, angiografia (Figura 2), tomografia computadorizada (Figura 3) e ressonância nuclear magnética podem ser usados tanto para confirmar o diagnóstico de TUCC quanto para seu seguimento. A abordagem inicial do primeiro paciente foi realizada por meio de angiotomografia e seu seguimento realizado por meio de ecodoppler. A segunda paciente foi 
diagnosticada e acompanhada por meio de ecodoppler. No caso do terceiro paciente, optou-se por uma investigação inicial com ressonância nuclear magnética e angiotomografia por se tratar de um caso bilateral; seu seguimento também foi realizado com ecodoppler.

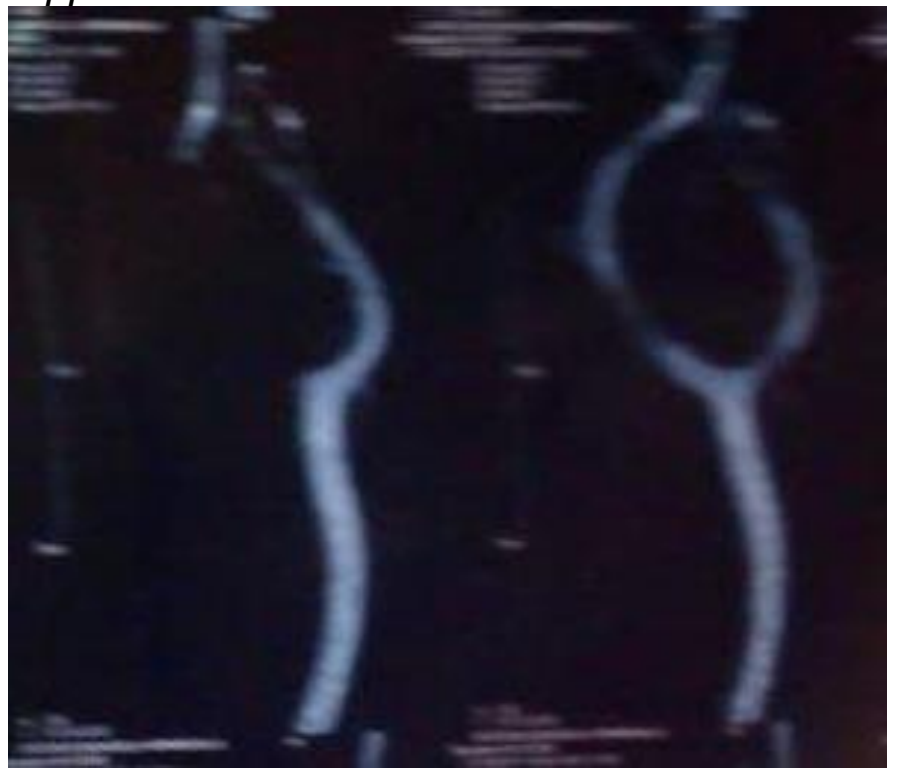

Figura 2 - Angiografia mostrando carótida interna e externa em volta do tumor (Fonte: prontuário do paciente).

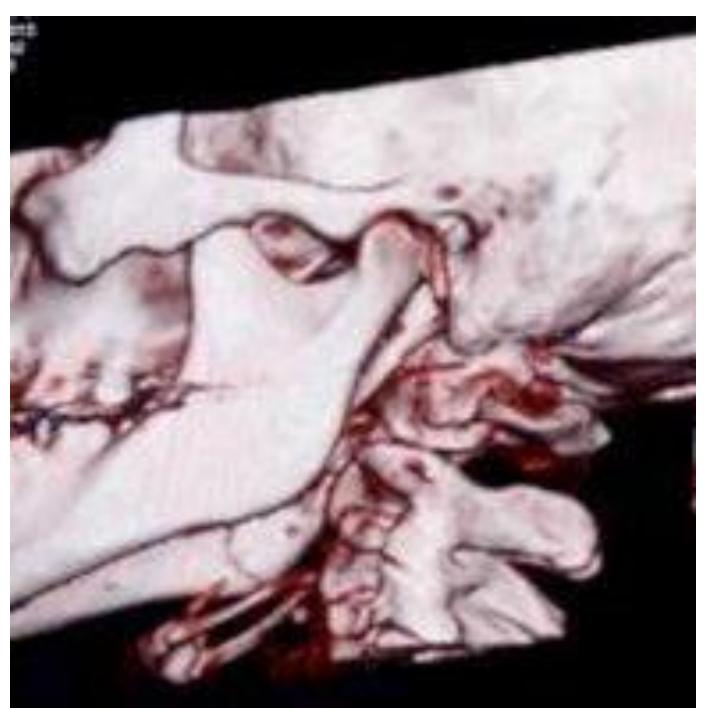

Figura 3 - Angiotomografia computadorizada pós-ressecção de lesão. (Fonte: prontuário do paciente).

O tratamento de escolha é a ressecção cirúrgica. Em 1971, Shamblin e colaboradores elaboraram uma classificação em três grupos diferentes para TUCC: Grupo 1 - pequenos tumores que não envolvem a carótida e de fácil ressecção; Grupo 2 - tumores maiores que envolvem parcialmente a carótida e possuem ressecção difícil; Grupo 3 - tumores de grandes dimensões que acomete toda a carótida e ressecção perigosa (SHAMBLIN et al, 1971). Os três pacientes descritos no caso se enquadravam no Grupo 2 de Shamblin e foram submetidos a procedimento cirúrgico para retirada do tumor. 


\section{CONCLUSÃO}

O diagnóstico de TUCC deve ser considerado em todo paciente que apresenta como queixa um crescimento ou tumefação em região de triângulo cervical do pescoço. Anamnese e exame físico já levam ao provável diagnóstico que poderá ser confirmado via exame de imagem. O preparo do paciente e mapeamento da lesão para intervenção cirúrgica é imprescindível para que se possa obter um resultado satisfatório com mínima ou nenhuma sequela para o paciente.

\section{REFERÊNCIAS}

ISIK AC, Imamoglu M, Erem C, Sari A. Paragangliomas of the head and neck. Med Princ Pract. 16(3):209-14, 2007.

KOTELIS, Drosos et al. Late outcome after surgical management of carotid body tumors from a 20-year single-center experience. Langenbeck's archives of surgery, v. 394, n. 2, p. 339-344, 2009.

MESQUITA, Nelson et al. Tumor de corpo carotídeo (paraganglioma): relato de dois casos submetidos a tratamento cirúrgico. Jornal vascular brasileiro, v. 15, n. 2, p. 158, 2016.

SAJID, M. S.; HAMILTON, G.; BAKER, D. M. A multicenter review of carotid body tumour management. European Journal of Vascular and Endovascular Surgery, v. 34, n. 2, p. 127-130, 2007. DOI: 10.1016/j.ejvs.2007.01.015

SHAMBLIN, William R. et al. Carotid body tumor (chemodectoma): clinicopathologic analysis of ninety cases. The American Journal of Surgery, v. 122, n. 6, p. 732739, 1971.

SOUSA, Atos Alves de et al. Tumores do corpo carotídeo: revisão de oito casos e abordagem cirúrgica. Arq. neuropsiquiatr, v. 58, n. 2A, p. 315-23, 2000.

VIEIRA, Leonardo José et al. Tumor de corpo carotídeo: relato de caso. Rev Med Minas Gerais, v. 19, n. 1, p. 71-74, 2009. 